\title{
Determination of volumetric concentration of solids in vertical pipeline hydrotransport
}

\author{
Marcin Zych ${ }^{1, a}$, Robert Hanus ${ }^{2}$, Leszek Petryka ${ }^{1}$, Anna Strzępowicz ${ }^{1}$ and Piotr Zych ${ }^{3}$ \\ ${ }^{1}$ A GH University of Science and Technology, Krakow 30-059, Poland \\ ${ }^{2}$ Rzeszow University of Technology, Rzeszow 35-959, Poland \\ ${ }^{3}$ Warsaw University of Technology, Warszawa 00-661, Poland
}

\begin{abstract}
This paper presents an application of radioisotopes with reference to the determination of the solid phase volumetric concentration in a hydromixture by calibration of the measuring set. It shows how the gamma absorption equipment consisting of radioactive isotopes ${ }^{241} \mathrm{Am}$ and scintillation probe, may be applied to the measurement of solid particles volumetric concentration in a flow. It is based on fact that the intensity of a gamma beam decreases as it passes through matter. In the described experiments as solid phase the ceramic models representing natural polymetallic ocean nodules were used. The especially constructed calibration stand and obtained relation between the related intensity of radiation and mean volumetric concentration of the solid phase are presented.
\end{abstract}

\section{Introduction}

Polymetallic nodules occur on the bottom of seas and oceans. They are solid structures of organic and mineral compositions which contain a various metals (mostly Mn, $\mathrm{Si}, \mathrm{Fe}, \mathrm{Al}, \mathrm{Na}, \mathrm{Mg}, \mathrm{Ni}, \mathrm{K}, \mathrm{Cu})$ [1-4]. The mining of nodules using the hydraulic method requires grain vertical transport by water. To determination of the solid phase volumetric flow rate in pipeline it is necessary to know the volumetric concentration of nodules and their velocity. For this purpose the method based on gamma ray absorption was proposed [5-10].

The idea of gamma-ray absorption measurement is presented in figure 1.

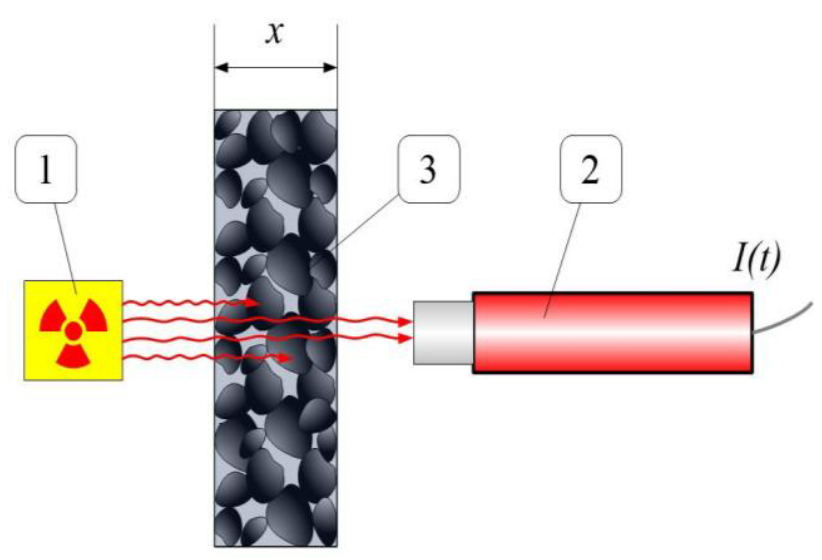

Figure 1. The principle of gamma radiation absorption measurement: 1 - gamma radiation source in collimator, 2 - scintillation probe, 3 - absorbent.
The gamma absorption is based on exponential decreasing of a monoenergetic gamma beam in function of composition and geometry of absorbent:

$$
I=I_{0} \cdot \exp (-\eta \cdot \mu \cdot x)
$$

where $I_{0}$ is the inlet to absorbent radiation intensity, $I$ is the outlet intensity detected after the beam has traveled a distance $x$ through the absorbing materials, $\eta$ and $\mu$ represents consequently the density and mass absorption coefficient of these materials [11-14].

The sealed radioactive sources emit gamma radiation beams shaped by collimators (1). Photons pass through the pipeline with analyzed mixture (3) and are achieved by the detector (2). In the presented experiment linear ${ }^{241} \mathrm{Am}$ source with energy of $59.5 \mathrm{keV}$ and probe with $\mathrm{NaI}(\mathrm{Tl})$ scintillation detectors are used.

Using two sets of source - probe presented in figure 1 we can measure the averaged velocity of the solid phase [6-10]. At the same time one of these sets can be used to determination of the volumetric concentration of solid particles.

In this work we use data recorded on the experimental laboratory stand built up in the Water Laboratory of the Wroclaw University of Environmental and Life Sciences (Poland) for investigation of the polymetallic nodules hydrotransport in a vertical pipe with a inner diameter $150 \mathrm{~mm}$. In the described experiments as solid phase the ceramic models representing three grain sizes (up to 70 $\mathrm{mm}$ ) of natural polymetallic ocean nodules was used. The density of the particles representing the nodules was 2000 $\mathrm{kg} / \mathrm{m}^{3}$.

The ceramic models of nodules are shown in figure 2.

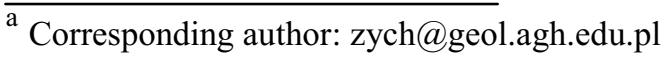




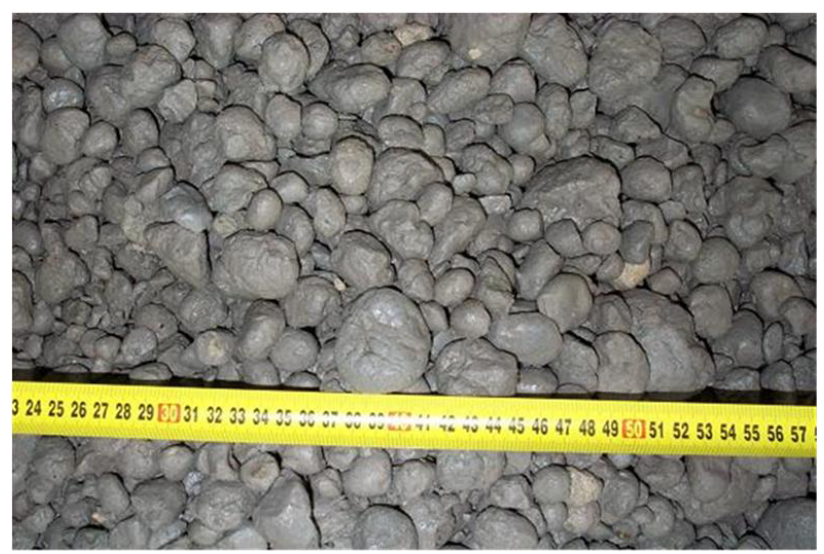

Figure 2. The ceramic models of ocean nodules used in experiments.

\section{Determination of volumetric concen- tration of solid particles}

The volumetric concentration in liquid-solid particles flow is defined as the ratio of the solid phase volume to the total volume of the mixture:

$$
C_{V}=\frac{V_{S}}{V}=\frac{V_{S}}{V_{W}+V_{S}}
$$

where $V_{W}$ is water volume, $V_{S}=V_{S 1}+V_{S 2}+\ldots$ is the sum of the volume of solid particles in the analyzed section $d z$ of the pipe. Figure 3 presents the essential parameters for determination of volume concentration.

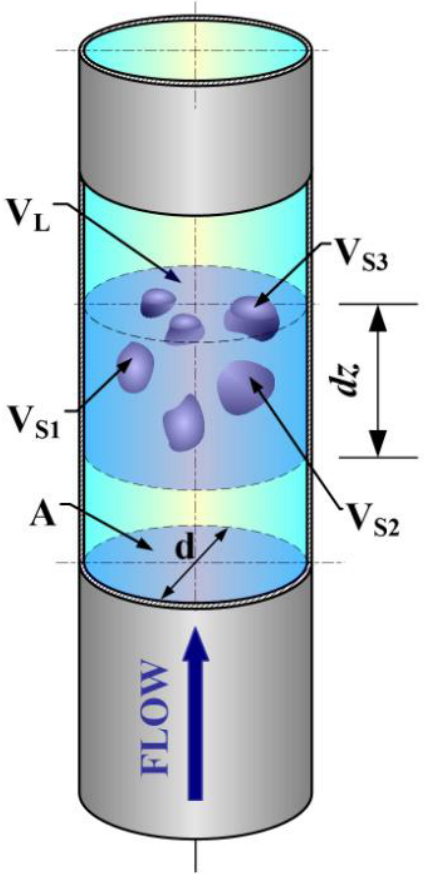

Figure 3. The essential parameters for determination of volume concentration.

Passing to the limit $d z \rightarrow 0$, the volumetric concentration of cross-section is defined as [15]:

$$
C_{V P}=\frac{A_{S}}{A}=\frac{A_{S}}{A_{W}+A_{S}}
$$

where: $A=\pi \cdot d^{2} / 4-$ cross sectional surface of the pipe, $d$ - inner diameter of the pipe, $A_{S}$ and $A_{W}$ - cumulative cross sectional surfaces of the solid phase and liquid phase respectively.

The weakening of radiation in a mixture of concentration $C_{V P}$ transported through pipeline can be described by the differential equation:

$$
\frac{d I}{d C_{V P}}+\mu_{C} \cdot I=0
$$

where $\mu_{C}$ is the relative volumetric absorption coefficient. For the boundary conditions that occur during the measurement the solution of equation (4) is:

$$
I=I_{0} \cdot \exp \left(-\mu_{C} \cdot C_{V P}\right)
$$

where $I_{0}$ is the intensity of radiation recorded by the probe, where the pipe is filled with water only $\left(C_{V P}=0\right)$.

Because it is difficult to consider all the phenomena of absorption of radiation and its detection, experimental calibration on the laboratory stand is necessary.

\section{Experimental setup and calibration procedure}

Determination of the relationship between concentration and intensity of gamma radiation weakened in the liquidsolid particles mixture was carried out on an experimental calibration stand built in the Sedimentological Laboratory in Faculty of Geology, Geophysics and Environmental Protection AGH University of Science and Technology in Krakow. Diagram of the calibration stand is shown in figure 4 .

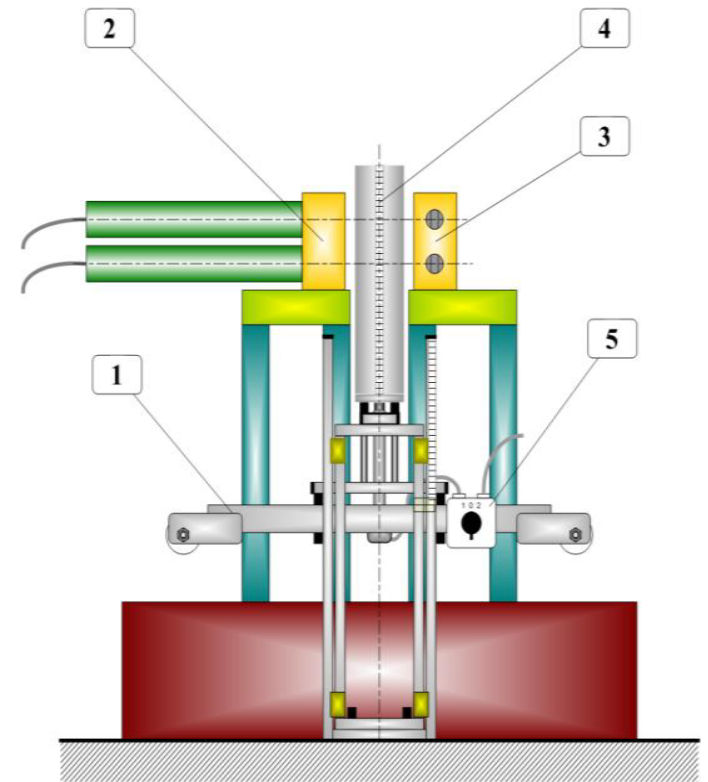

Figure 4. Diagram of the calibration stand: 1 - universal trolley, 2 - collimator with scintillation probes, 3 - lead collimator with gamma ray sources, 4 - calibration section of the pipeline, 5 - switch to control of cross-section high. 
The stand consists of a universal measuring trolley (1) with the possibility of vertical travel, the controlled switch (5) and the calibration section of the pipeline (4). The fragment containing the test pipe with liquid-solids mixture was placed in the same way as during the nodule hydrotransport measurements. The gamma beams of radiation formed through collimator (3) and (2) pass through the pipeline at a predetermined height.

The mixture was prepared in such way that in the test section of pipe a real nodules concentration was reconstructed:

- for $C_{V P}$ range of $0.400 \div 0.700$ models filled the entire volume of the pipe,

- for $C_{V P}$ range of $0.180 \div 0.400$ the additional thinwalled plastic container with dimensions of $110 \mathrm{~mm} \times$ $120 \mathrm{~mm} \times 200 \mathrm{~mm}$ was used,

- for $C_{V P}$ range of $0.010 \div 0.180$ the fragment of the cone with diameters of $\varnothing 80$ to $85 \mathrm{~mm}$ and a height of $250 \mathrm{~mm}$ was used.

In order to determine the concentration, the pipe was progressively filled with water. The thickness of each layer of water was measured by using a laser range finder and caliper gauge. During refilling with water, the test section of the pipeline was placed on the scale, which allowed measurement of the mass increase of a further layer of the liquid.

During calibration the volumetric concentration of the solid phase $C_{V P}$ was determined from the relationship:

$$
C_{V P}=1-C_{V W}
$$

where

$$
C_{V W}=\frac{4 \cdot m_{W}}{\rho_{W} \cdot \pi \cdot d^{2} \cdot d z}
$$

where $m_{W}$ is the weight of the respective water layer having a thickness $d z$, and $\rho_{W}$ is density of water.

For each layer a series of 18000 measurements of radiation intensity was made. Each time the background radiation was measured before and after experiment. To properly map the distribution of solid particles in the pipeline, container was additionally rotated of angles from $45^{\circ}$ to $180^{\circ}$ during the measurement.

As a result of the study the following calibration curve was obtained using a linear least squares fitting technique:

$$
\ln \left(\frac{I}{I_{0}}\right)=a \cdot C_{V P}+b
$$

where regression coefficients are: $a=-2.58 \pm 0.06$ and $b=-0.23 \pm 0.03$ at coefficient of determination $R^{2}=0.99$.

The graph of the obtained straight line is shown in figure 5 .

Dependence (8) allows to determining the average volume concentration of solid phase in nodules hydrotransport measurements conducted at the research installation.

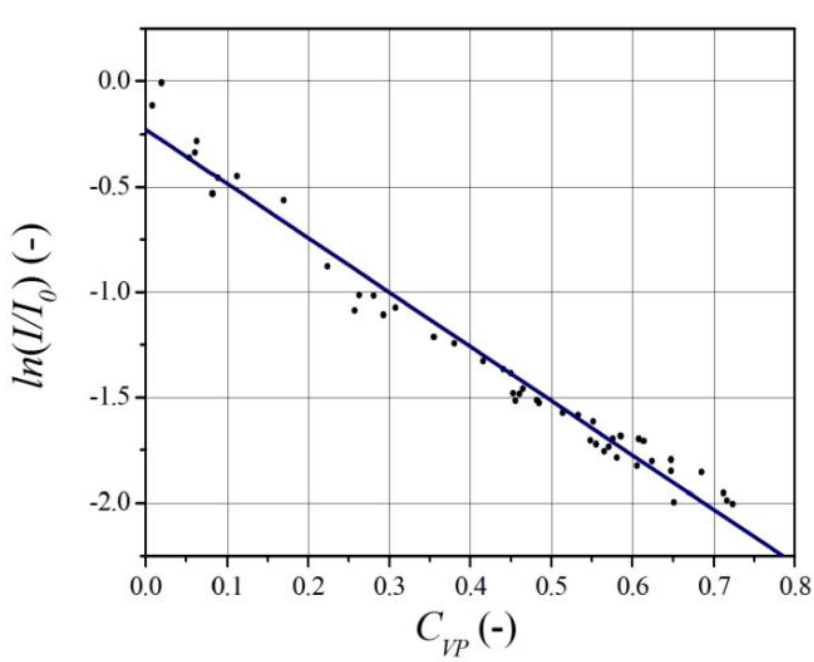

Figure 5. The relation between $I / I_{0}$ and the volumetric concentration $C_{V P}$.

\section{Exemplary results}

To use the calibration curve in practice, they are essential reference measurements, which are carried out to the water itself. This allows to find the point for which the value $C_{V P}=0$. Thus this enables temperature compensation and taking into account the stability of the apparatus. It should be noted that the slope of the function is unchanged, because it is associated solely with the composition of the transported mixture.

The curve shown in figure 5 were used in the studies of hydrotransport of ceramic models of polymetallic nodules conducted in the Water Laboratory of the Wroclaw University of Environmental and Life Sciences. For example, figure 6 presents the results obtained in the experiment WRS039. The line with points shows the evolution of the concentration of the solid phase for the averaging in the time interval of 10 seconds. Whereas the horizontal straight line shows the average value for the whole measurement (300 seconds).

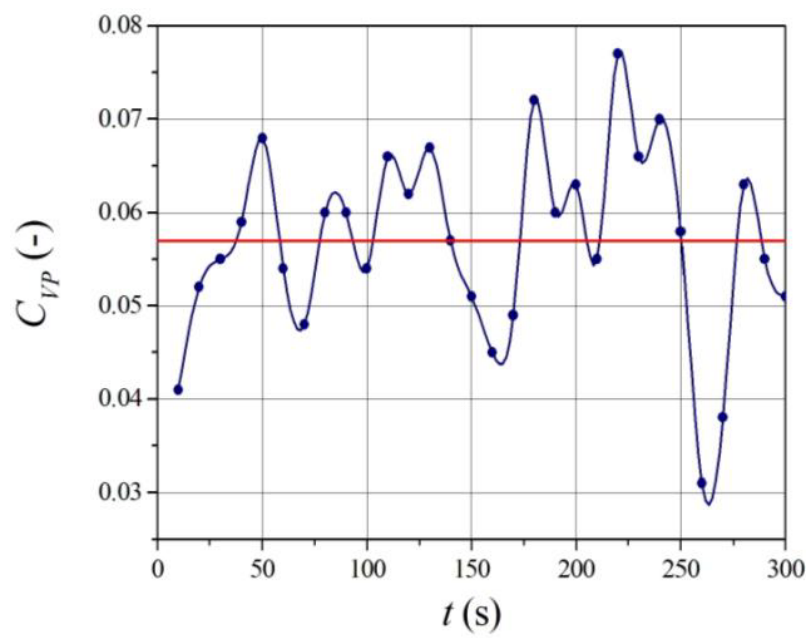

Figure 6. Changes of the $C_{V P}$ value averaged in time interval of 10 seconds (line with points) and an average $C_{V P}$ value for the whole measurement (horizontal straight line) in experiment WRS039. 
The average value $C_{V P}$ for the whole measurement presented in figure 6 is equal to $0.057 \pm 0.003$. The $C_{V P}$ values averaged in time interval of 10 seconds are characterized by relatively high volatility in the range from 0.030 to 0.080 . This means that the grains of the solid phase not flowed continuously but formed clusters, between which there was only water.

The measurement accuracy of $C_{V P}$ was determined with law of propagation of uncertainty [16].

In the case of use of the calibration line the fluctuations in the intensity of radiation $I$ should be considered. They are the result of the statistical nature of radioactive decay and are described by the standard uncertainty [17]:

$$
u(I)=\sqrt{I}
$$

On the basis of the analysis we can be stated that the uncertainty of the average volumetric concentration of solid phase in the flowing mixture does not exceed $10 \%$.

\section{Conclusions}

For determination of volumetric concentration $C_{V P}$ using gamma absorption it is necessary to calibrate a measuring set of source of radiation - scintillation probe.

Implementation of the calibration procedure required to build a laboratory stand for which reconstruction of fragment of pipeline and modeling of the flow structures were conducted. The resulting calibration dependence allows the determination of the average volumetric concentration of solid phase for the measured radiation intensity. On the basis of the $C_{V P}$ value and the velocity of the solid phase we can determine the volumetric flow rate of the nodules [15].

The advantage of the presented solution is to use the same absorption set to measure of both: the volumetric concentration of the solid phase and their average velocity [6-10]. This allows the determination of the volumetric flow rate of the solid phase additional detectors or independent measurements.

Described in the paper calibration procedure can be in the future replaced by simulations, using e.g. MCNP software.

\section{Acknowledgment}

The authors would like to thank teams of prof. Jerzy Sobota and prof. Pavel Vlasak for their cooperation during the measurements undertaken at Wroclaw University of Environmental and Life Sciences.

All presented investigations were possible due to support under the project P105/10/1574 of the Grant Agency of the Czech Republic, and RVO: 67985874 of the ASCR, as well as the Project No NN523755340 of the National Science Centre of Poland, which are gratefully acknowledged.

This publication is funded by $\mathrm{AGH}$ University of Science and Technology (No 11.11.140.645).

\section{References}

1. D.S. Cronan (ed.), Handbook of marine mineral deposits (CRC Press, Boca Raton, 2000)

2. R. Kotliński, http://www.iom.gov.pl/uploads/iom attachment-95635.pdf

3. S. Depowski, et al, Mineral resources of the seas and oceans (SCHOLAR, Warsaw, 1998)

4. http://www.iom.gov.pl/

5. L. Petryka, M. Zych, R. Murzyn, Nukleonika 50, 43-46 (2005)

6. R. Hanus, L. Petryka, M. Zych, Flow Meas. Instrum. 40, 58-63 (2014)

7. L. Petryka, et al, EPJ WoC 92, 02065 (2015)

8. R. Hanus, et al, EPJ WoC 92, 02020 (2015)

9. R. Hanus, et al., Math. Probl. Eng. 2014, 475735 (2014)

10. L. Petryka, et al, Proc. 10th ISOPE Ocean Mining \& Gas Hydrates Symp. OMS-2013, 230-233 (2013)

11. G. Falcone, G.F. Hewitt, C. Alimonti, Multiphase flow metering: principles and applications (Elsevier, Amsterdam, 2009)

12. G.A. Johansen, P. Jackson, Radioisotope gauges for industrial process measurements (Wiley, New York, 2004)

13. M. Zych, et al., Flow Meas. Instrum. 35, 11-15 (2014)

14. Z. Yu, B. Qincheng, H. Richa, Appl. Therm. Eng. 60, 398-410 (2013)

15. M. Zych, et al., MAaM 56 (4), 318-321 (2010)

16. Guide to the expression of uncertainly in measurement (International Organisation for Standardisation, 1995)

17. G.F Knoll, Radiation detection and measurement (Wiley, New York, 1986) 